\title{
Large-Scale Phenotype-Based Antiepileptic Drug Screening in a Zebrafish Model of Dravet Syndrome $e^{1,2,3}$
}

\author{
Matthew T. Dinday, ${ }^{1}$ and Scott C. Baraban ${ }^{1,2}$
}

DOI:http://dx.doi.org/10.1523/ENEURO.0068-15.2015

${ }^{1}$ Department of Neurological Surgery, Epilepsy Research Laboratory, University of California San Francisco, San Francisco, California 94143, ${ }^{2}$ Eli and Edythe Broad Center of Regeneration Medicine and Stem Cell Research, University of California San Francisco, San Francisco, California 94143

\begin{abstract}
Mutations in a voltage-gated sodium channel (SCN1A) result in Dravet Syndrome (DS), a catastrophic childhood epilepsy. Zebrafish with a mutation in scn1Lab recapitulate salient phenotypes associated with DS, including seizures, early fatality, and resistance to antiepileptic drugs. To discover new drug candidates for the treatment of DS, we screened a chemical library of $\sim 1000$ compounds and identified 4 compounds that rescued the behavioral seizure component, including 1 compound (dimethadione) that suppressed associated electrographic seizure activity. Fenfluramine, but not huperzine A, also showed antiepileptic activity in our zebrafish assays. The effectiveness of compounds that block neuronal calcium current (dimethadione) or enhance serotonin signaling (fenfluramine) in our zebrafish model suggests that these may be important therapeutic targets in patients with DS. Over 150 compounds resulting in fatality were also identified. We conclude that the combination of behavioral and electrophysiological assays provide a convenient, sensitive, and rapid basis for phenotype-based drug screening in zebrafish mimicking a genetic form of epilepsy.
\end{abstract}

Key words: antiepileptic; drug discovery; epilepsy; high throughput; pharmacology; zebrafish

\section{Significance Statement}

Dravet syndrome is a catastrophic childhood epilepsy that is resistant to available medications. Current animal models for this disease are not amenable to high-throughput drug screening. We used a zebrafish model for Dravet syndrome and screened $>1000$ compounds. We report the identification of compounds with the ability to suppress seizure behavior and electrographic seizure activity. This approach provides an example of precision medicine directed to pediatric epilepsy.

\section{Introduction}

Dravet syndrome (DS) is a devastating genetic epileptic encephalopathy that has been linked to more than $>300$

Received June 18, 2015; accepted August 4, 2015; First published August 20, 2015.

${ }^{1}$ The authors declare no competing financial interests.

${ }^{2}$ Author contributions: M.T.D. and S.C.B. designed research; M.T.D. and S.C.B. performed research; M.T.D. and S.C.B. analyzed data; S.C.B. wrote the paper.

${ }^{3}$ Funding was provided by National Institutes of Health-National Institute of Neurological Disorders and Stroke EUREKA Grant 5R01-NS-079214 and The Joseph \& Vera Long Foundation to (S.C.B.). de novo mutations in a neuronal voltage-gated sodium channel $(S C N)$. Children with DS are at a higher risk for sudden unexplained death in epilepsy and episodes of

Acknowledgments: We thank B. Grone and D. Lowenstein for comments on earlier versions of this manuscript.

Correspondence should be addressed to Scott C. Baraban, Department of Neurological Surgery, Epilepsy Research Laboratory, University of California, San Francisco, San Francisco, CA 94143. E-mail: scott.baraban@ucsf.edu. DOl:http://dx.doi.org/10.1523/ENEURO.0068-15.2015

Copyright (C) 2015 Dinday and Baraban

This is an open-access article distributed under the terms of the Creative Commons Attribution 4.0 International, which permits unrestricted use, 
uncontrolled status epilepticus (Dravet et al., 2005; Ceulemans et al., 2012). Delayed language development, disruption of autonomic function, and motor and cognitive impairment are also associated with this disease. Seizure management includes treatment with benzodiazepines, valproate, and/or stiripentol (Caraballo et al., 2005; Chiron and Dulac, 2011). Some reduction in seizure activity has been reported with the use of bromides and topiramate, or a ketogenic diet (Lotte et al., 2012; Wilmshurst et al., 2014; Dressler et al., 2015). Despite these options, available antiepileptic drugs (AEDs) do not achieve adequate seizure control in most DS patients (Dravet et al., 2005; Chiron and Dulac, 2011; Dressler et al., 2015), making the identification of new drugs a critical unmet need. Highthroughput screening offers a powerful tool to identify new drug candidates for these patients. However, commonly available screening approaches rely on in vitro cell-based assays (Masimirembwa et al., 2001; Snowden and Green, 2008; Ko and Gelb, 2014) and do not recapitulate the complicated neural networks that generate seizures in vivo. Given the need for new treatments for children with DS, and the growing number of genetic epileptic encephalopathies that are medically intractable (Leppert, 1990; Epi4K Consortium, 2012; Ottman and Risch, 2012), we developed an alternative phenotypebased in vivo drug-screening strategy. While cell-based in vitro screening assays can efficiently identify compounds that bind specific targets, whole-organism-based screens are more likely to reliably predict therapeutic outcomes as they maintain the complex neural circuitry involved in the underlying disease process. Whole-organism screens do not require well validated targets to identify compounds that yield a desirable phenotypic outcome, but can be prohibitively costly and time consuming in mammals. As a simple vertebrate with significant genetic similarity to human, zebrafish are now recognized as an ideal costeffective alternative to achieve rapid in vivo phenotypebased screening (Ali et al., 2011).

Using scn1a mutant zebrafish larvae with a gene homologous to human and spontaneously occurring seizures (Baraban et al., 2013), we screened, in a blinded manner, a repurposed library of $\sim 1000$ compounds for drugs that inhibit unprovoked seizure events. We also screened two compounds (huperzine A and fenfluramine) that were discovered in rodent-based assays using acquired seizure protocols and that were recently suggested as potential treatments for DS (Boel and Casaer, 1996; Coleman et al., 2008; Ceulemans et al., 2012; Bialer et al., 2015). Only 20 compounds in the repurposed drug library reduced swim behavior to control levels. However, many of these compounds were toxic or were not confirmed on retesting, and only four compounds advanced to a second-stage in vivo electrophysiology assay. Of these compounds (cytarabine, dimethadione, theobromine, and norfloxacin) only dimethadione, a T-type calcium channel antagonist previously reported to have anticonvulsant activity (Lowson et al., 1990; Zhang et al., 1996), reduced

distribution and reproduction in any medium provided that the original work is properly attributed. ictal-like electrographic discharges seen in scn1Lab mutant larvae. This two-stage phenotype-based screening approach, using a genetic DS model with $>75 \%$ genomic similarity to human, is a sensitive, rapid means to successfully identify compounds with antiepileptic activity.

\section{Materials and Methods}

\section{Zebrafish}

Zebrafish were maintained in a light- and temperaturecontrolled aquaculture facility under a standard 14:10 h light/dark photoperiod. Adult zebrafish were housed in 1.5 $\mathrm{L}$ tanks at a density of 5-12 fish per tank and fed twice per day (dry flake and/or flake supplemented with live brine shrimp). Water quality was continuously monitored: temperature, $28-30^{\circ} \mathrm{C}$; $\mathrm{pH}$ 7.4-8.0; conductivity, 690-710 $\mathrm{mS} / \mathrm{cm}$. Zebrafish embryos were maintained in round $\mathrm{Pe}-$ tri dishes (catalog \#FB0875712, Fisher Scientific) in "embryo medium" consisting of $0.03 \%$ Instant Ocean (Aquarium Systems, Inc.) and 000002\% methylene blue in reverse osmosis-distilled water. Larval zebrafish clutches were bred from wild-type (WT; TL strain) or scn1Lab (didy ${ }^{\mathrm{s5} 2}$ ) heterozygous animals that had been backcrossed to TL wild-type for at least 10 generations. Homozygous mutants $(n=6544)$, which have widely dispersed melanosomes and appear visibly darker as early as $3 \mathrm{~d}$ postfertilization (dpf; Fig. 1b), or WT larvae ( $n$ $=71$ ) were used in all experiments at 5 or $6 \mathrm{dpf}$. Embryos and larvae were raised in plastic petri dishes $(90 \mathrm{~mm}$ diameter, $20 \mathrm{~mm}$ depth) and density was limited to $\sim 60$ per dish. Larvae between 3 and 7 dpf lack discernible sex chromosomes. The care and maintenance protocols comply with requirements outlined in the Guide for the Care and Use of Animals (ebrary Inc., 2011) and were approved by the Institutional Animal Care and Use Committee (protocol \#AN108659-01D).

\section{Seizure monitoring}

Zebrafish larvae were placed individually into 1 well of a clear flat-bottomed 96-well microplate (catalog \#260836, Fisher Scientific) containing embryo media. Microplates were placed inside an enclosed motion-tracking device and acclimated to the dark condition for 10-15 min at room temperature. Locomotion plots were obtained for one fish per well at a recording epoch of 10 min using a DanioVision system running EthoVision XT software (DanioVision, Noldus Information Technology); threshold detection settings to identify objects darker than the background were optimized for each experiment. Seizure scoring was performed using the following three-stage scale (Baraban et al., 2005): Stage 0, no or very little swim activity; Stage I, increased, brief bouts of swim activity; Stage II, rapid "whirlpool-like" circling swim behavior; and Stage III, paroxysmal whole-body clonus-like convulsions, and a brief loss of posture. WT fish are normally scored at Stage 0 or I. Plots were analyzed for distance traveled (in millimeters) and mean velocity (in millimeters per second). As reported previously (Winter et al., 2008; Baraban et al., 2013), velocity changes were a more sensitive assay of seizure behavior. For electrophysiology studies, zebrafish larvae were briefly paralyzed with 


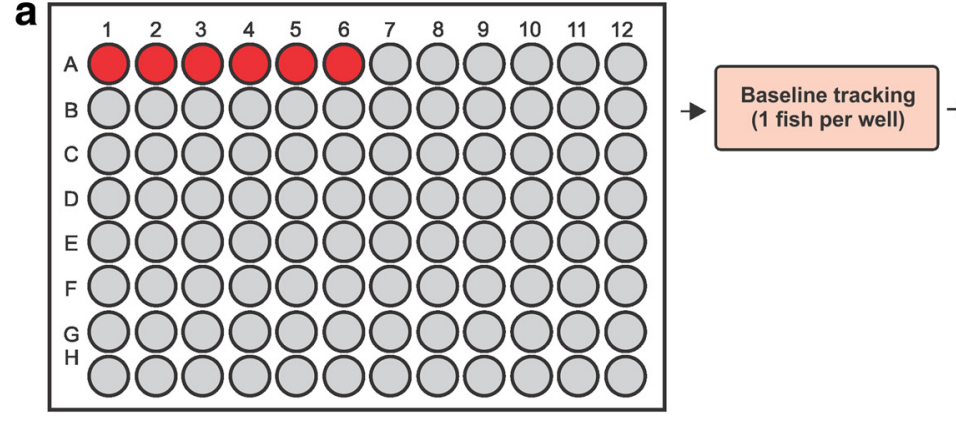

b
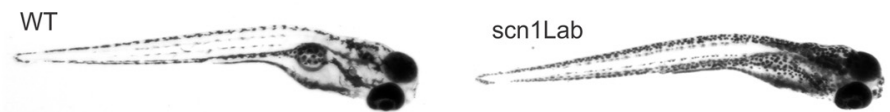

d

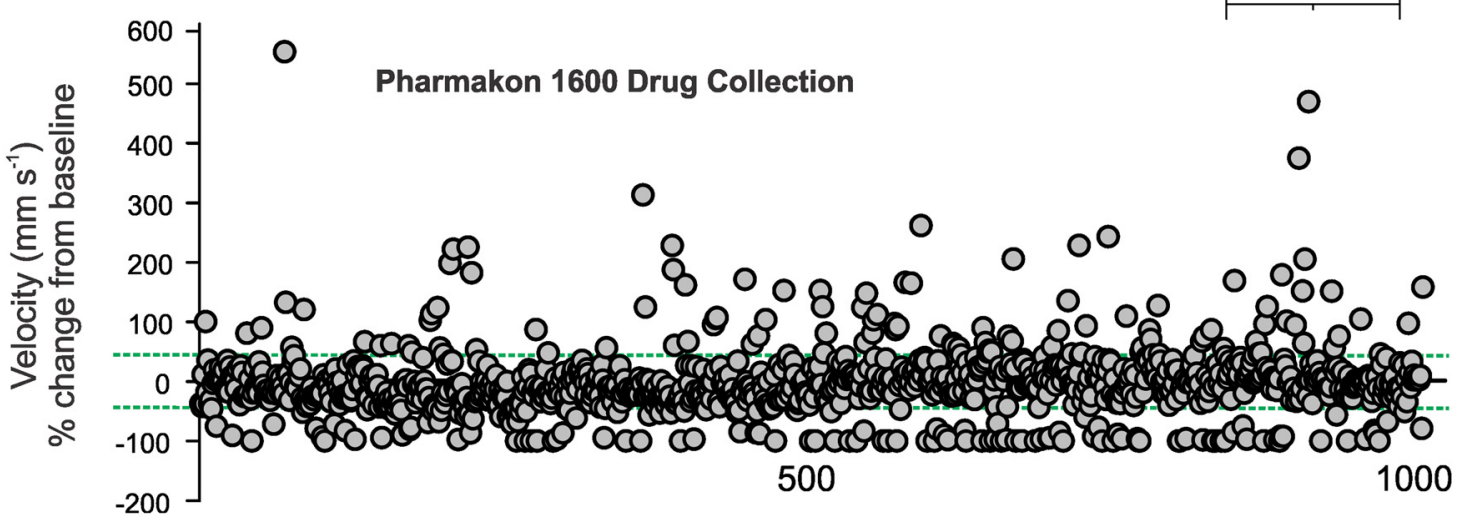

Figure 1. Locomotion assay to identify drugs that rescue the scn1Lab mutant epilepsy phenotype. a, Schematic of the phenotypebased screening process. Chemical libraries can be coded and aliquoted in small volumes (75 $\mu \mathrm{L})$ into individual wells containing one mutant fish. The 96-well microplate is arranged so that six fish are tested per drug; with one row of six fish maintained as an internal control (red circles) on each plate. $\boldsymbol{b}$, Representative images for WT and scn1Lab mutant zebrafish larvae at 5 dpf. Note the morphological similarity but darker pigmentation in mutant larvae. $\boldsymbol{c}$, Box plot of mean velocity (in millimeters per second) for two consecutive recordings of mutant larvae in embryo media. Experiments were performed by first placing the mutant larvae in embryo media and obtaining a baseline locomotion response; embryo media was then replaced with new embryo media (to mimic the procedure used for test compounds), and a second locomotion response was obtained. The percentage change in velocity from baseline (recording 1) versus experimental model (recording 2) is shown. In the box plot, the bottom and top of the box represent the 25th percentile and the 75th percentile, respectively. The line across the box represents the median value, and the vertical lines encompass the entire range of values. This plot represents normal changes in tracking activity in the absence of a drug challenge. $\boldsymbol{d}$, Plot of locomotor seizure behavior for scn1 Lab mutants at $5 \mathrm{dpf}$ for the 1012 compounds tested. Threshold for inhibition of seizure activity (positive hits) was set as a reduction in mean swim velocity of $\geq 44 \%$; the threshold for a proconvulsant or hyperexcitable effect was set at an increase in the mean swim velocity of $\geq 44 \%$ (green dashed lines).

$\alpha$-bungarotoxin $(1 \mathrm{mg} / \mathrm{ml})$ and immobilized in $1.2 \%$ agarose; field recordings were obtained from forebrain structures. Epileptiform events were identified post hoc in Clampfit (Molecular Devices) and were defined as multispike or polyspike upward or downward membrane deflections greater than three times the baseline noise level and $>500 \mathrm{~ms}$ in duration. During electrophysiology experiments zebrafish larvae were continuously monitored for the presence (or absence) of blood flow and heart beat by direct visualization on an Olympus BX51WI upright microscope equipped with a CCD camera and monitor.

\section{Drugs}

Compounds for drug screening were purchased from MicroSource Discovery Systems, Inc. (PHARMAKON 1600 ) and were provided as $10 \mathrm{~mm}$ DMSO solutions (Table 1). Test compounds for locomotion or electrophysiology studies were dissolved in embryo media and were tested at an initial concentration of $100 \mu \mathrm{M}$, with a final DMSO concentration of $<2 \%$. In all drug library screen studies, compounds were coded and experiments were performed by investigators who were blind to the nature of the compound. Baseline recordings of seizure behavior 
Table 1. List of compounds from the PHARMAKON 1600 library used in this screen.

ABACAVIR SULFATE

ABAMECTIN (avermectin B1a shown)

ACADESINE

ACARBOSE

ACEBUTOLOL HYDROCHLORIDE

ACECLIDINE

ACECLOFENAC

ACENOCOUMAROL

ACETAMINOPHEN

ACETOHYDROXAMIC ACID

ACETOPHENAZINE MALEATE

ACETRIAZOIC ACID

ACETYLCHOLINE CHLORIDE

ACETYLCYSTEINE

ACIPIMOX

ACONITINE

ACRIFLAVINIUM HYDROCHLORIDE

ACRISORCIN

ACTARIT

ACYCLOVIR

ADAPALENE

ADELMIDROL

ADENINE

ADENOSINE

ADENOSINE PHOSPHATE

ADIPHENINE HYDROCHLORIDE

AKLOMIDE

ALAPROCLATE

ALBENDAZOLE

ALBUTEROL (+/-)

ALENDRONATE SODIUM

ALEXIDINE HYDROCHLORIDE

ALLANTOIN

ALLOPURINOL

ALMOTRIPTAN

alpha-TOCHOPHEROL

alpha-TOCHOPHERYL ACETATE

ALPRAZOLAM

ALRESTATIN

ALTHIAZIDE

ALTRETAMINE

ALVERINE CITRATE

AMANTADINE HYDROCHLORIDE

AMCINONIDE

AMIFOSTINE

AMIKACIN SULFATE

AMILORIDE HYDROCHLORIDE

AMINACRINE

AMINOCAPROIC ACID

AMINOGLUTETHIMIDE

AMINOHIPPURIC ACID

AMINOLEVULINIC ACID HYDROCHLORIDE

AMINOSALICYLATE SODIUM

AMITRIPTYLINE HYDROCHLORIDE

AMLEXANOX

AMLODIPINE BESYLATE

AMODIAQUINE DIHYDROCHLORIDE

AMOROLFINE HYDROCHLORIDE

AMOXICILLIN

AMPHOTERICIN B

AMPICILLIN SODIUM

(Continued)
Table 1. List of compounds from the PHARMAKON 1600 library used in this screen. (continued)

AMPROLIUM

AMSACRINE

ANASTROZOLE

ANCITABINE HYDROCHLORIDE

ANETHOLE

ANIRACETAM

ANISINDIONE

ANTAZOLINE PHOSPHATE

ANTHRALIN

ANTIPYRINE

APOMORPHINE HYDROCHLORIDE

APRAMYCIN

ARGININE HYDROCHLORIDE

ARMODAFINIL

ARTEMETHER

ARTEMOTIL

ARTESUNATE

ASCORBIC ACID

ASPIRIN

ATENOLOL

ATORVASTATIN CALCIUM

ATOVAQUONE

ATROPINE SULFATE

AUROTHIOGLUCOSE

AVOBENZONE

AZACITIDINE

AZASERINE

AZATADINE MALEATE

AZATHIOPRINE

AZELAIC ACID

AZITHROMYCIN

AZLOCILLIN SODIUM

AZTREONAM

BACAMPICILLIN HYDROCHLORIDE

BACITRACIN

BACLOFEN

BALSALAZIDE DISODIUM

BECLOMETHASONE DIPROPIONATE

BEKANAMYCIN SULFATE

BEMOTRIZINOL

BENAZEPRIL HYDROCHLORIDE

BENDROFLUMETHIAZIDE

BENORILATE

BENSERAZIDE HYDROCHLORIDE

BENZALKONIUM CHLORIDE

BENZETHONIUM CHLORIDE

BENZOCAINE

BENZOIC ACID

BENZONATATE

BENZOYL PEROXIDE

BENZTHIAZIDE

BENZYL ALCOHOL

BENZYL BENZOATE

BEPRIDIL HYDROCHLORIDE

BERGAPTEN

beta-CAROTENE

BETAHISTINE HYDROCHLORIDE

BETAINE HYDROCHLORIDE

BETAMETHASONE

BETAMETHASONE 17,21-DIPROPIONATE BETAMETHASONE VALERATE

(Continued) 
Table 1. List of compounds from the PHARMAKON 1600 library used in this screen. (continued)

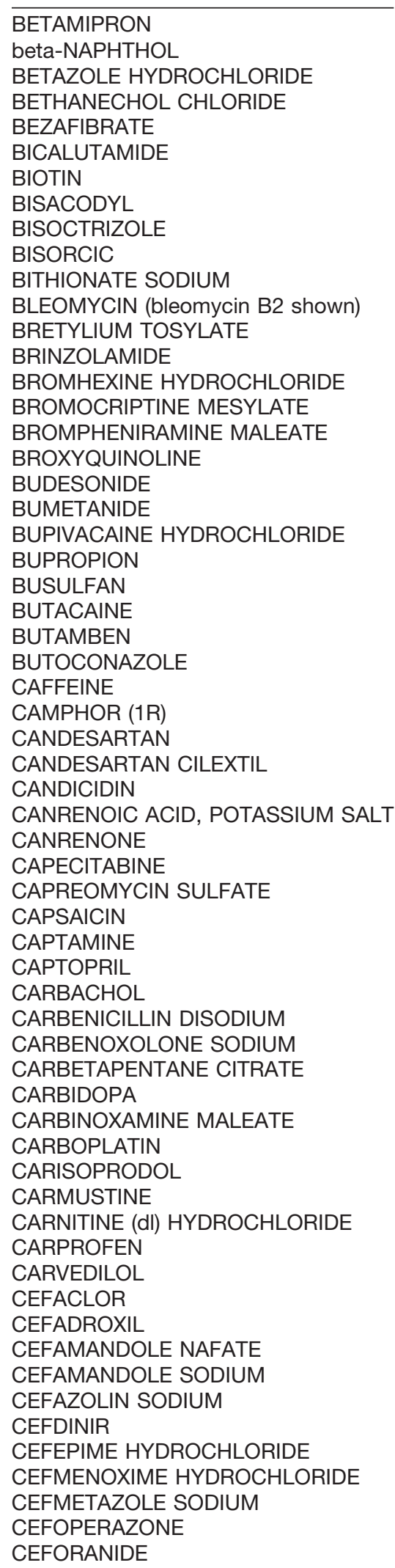

Table 1. List of compounds from the PHARMAKON 1600 library used in this screen. (continued)

CEFOTAXIME SODIUM CEFOTETAN

CEFOXITIN SODIUM

CEFPIRAMIDE

CEFSULODIN SODIUM

CEFTIBUTEN

CEFTIOFUR HYDROCHLORIDE

CEFTRIAXONE SODIUM TRIHYDRATE

CEFUROXIME AXETIL

CEFUROXIME SODIUM

CELECOXIB

CEPHALEXIN

CEPHALOTHIN SODIUM

CEPHAPIRIN SODIUM

CEPHRADINE

CETYLPYRIDINIUM CHLORIDE

CHENODIOL

CHLORAMBUCIL

CHLORAMPHENICOL

CHLORAMPHENICOL HEMISUCCINATE

CHLORAMPHENICOL PALMITATE

CHLORCYCLIZINE HYDROCHLORIDE

CHLORHEXIDINE

CHLOROCRESOL

CHLOROGUANIDE HYDROCHLORIDE

CHLOROQUINE DIPHOSPHATE

CHLOROTHIAZIDE

CHLOROXINE

CHLOROXYLENOL

CHLORPHENIRAMINE (S) MALEATE

CHLORPROMAZINE

CHLORPROPAMIDE

CHLORPROTHIXENE HYDROCHLORIDE

CHLORTETRACYCLINE HYDROCHLORIDE

CHLORTHALIDONE

CHLORZOXAZONE

CHOLECALCIFEROL

CHOLESTEROL

CHOLINE CHLORIDE

CICLOPIROX OLAMINE

CILOSTAZOL

CIMETIDINE

CINCHOPHEN

CINNARAZINE

CINOXACIN

CINTRIAMIDE

CIPROFIBRATE

CIPROFLOXACIN

CISPLATIN

CITALOPRAM HYDROBROMIDE

CITICOLINE

CLARITHROMYCIN

CLAVULANATE LITHIUM

CLEMASTINE

CLIDINIUM BROMIDE

CLINAFOXACIN HYDROCHLORIDE

CLINDAMYCIN HYDROCHLORIDE

CLIOQUINOL

CLOBETASOL PROPIONATE

CLOFARABINE

CLOFIBRATE

(Continued) 
Table 1. List of compounds from the PHARMAKON 1600 library used in this screen. (continued)

CLOMIPHENE CITRATE

CLONIDINE HYDROCHLORIDE

CLOPIDOGREL SULFATE

CLORSULON

CLOSANTEL

CLOTRIMAZOLE

CLOXACILLIN SODIUM

CLOXYQUIN

CLOZAPINE

COENZYME B12

COLCHICINE

COLFORSIN

COLISTIMETHATE SODIUM

CORTISONE ACETATE

COTININE

CRESOL

CROMOLYN SODIUM

CRYOFLURANE

CYCLAMIC ACID

CYCLIZINE

CYCLOBENZAPRINE HYDROCHLORIDE

CYCLOHEXIMIDE

CYCLOPENTOLATE HYDROCHLORIDE

CYCLOPHOSPHAMIDE HYDRATE

CYCLOSERINE (D)

CYCLOSPORINE

CYCLOTHIAZIDE

CYPERMETHRIN

CYPROTERONE ACETATE

CYSTEAMINE HYDROCHLORIDE

CYTARABINE

DACARBAZINE

DACTINOMYCIN

DANAZOL

DANTHRON

DANTROLENE SODIUM

DAPSONE

DAPTOMYCIN

DASATINIB

DAUNORUBICIN

DECIMEMIDE

DEFEROXAMINE MESYLATE

DEFLAZACORT

DEHYDROACETIC ACID

DEHYDROCHOLIC ACID

DEMECLOCYCLINE HYDROCHLORIDE

DERACOXIB

DESIPRAMINE HYDROCHLORIDE

DESOXYCORTICOSTERONE ACETATE

DESVENLAFAXINE SUCCINATE

DEXAMETHASONE

DEXAMETHASONE ACETATE

DEXAMETHASONE SODIUM PHOSPHATE

DEXIBUPROFEN

DEXLANSOPRAZOLE

DEXPROPRANOLOL HYDROCHLORIDE

DEXTROMETHORPHAN HYDROBROMIDE DIAVERIDINE

DIBENZOTHIOPHENE

DIBUCAINE HYDROCHLORIDE

DICHLOROPHENE

(Continued)
Table 1. List of compounds from the PHARMAKON 1600 library used in this screen. (continued)

DICHLORVOS

DICLAZURIL

DICLOFENAC SODIUM

DICLOXACILLIN SODIUM

DICUMAROL

DICYCLOMINE HYDROCHLORIDE

DIENESTROL

DIETHYLCARBAMAZINE CITRATE

DIETHYLSTILBESTROL

DIFLOXACIN HYDROCHLORIDE

DIFLUNISAL

DIGITOXIN

DIGOXIN

DIHYDROERGOTAMINE MESYLATE

DIHYDROSTREPTOMYCIN SULFATE

DILAZEP DIHYDROCHLORIDE

DIMENHYDRINATE

DIMERCAPROL

DIMETHADIONE

DIOXYBENZONE

DIPHENHYDRAMINE HYDROCHLORIDE

DIPHENYLPYRALINE HYDROCHLORIDE

DIPYRIDAMOLE

DIPYRONE

DIRITHROMYCIN

DISOPYRAMIDE PHOSPHATE

DISULFIRAM

DOBUTAMINE HYDROCHLORIDE

DOCETAXEL

DONEPEZIL HYDROCHLORIDE

DOPAMINE HYDROCHLORIDE

DOXEPIN HYDROCHLORIDE

DOXOFYLLINE

DOXYCYCLINE HYDROCHLORIDE

DOXYLAMINE SUCCINATE

DROFENINE HYDROCHLORIDE

DROPERIDOL

DROSPIRENONE

DYCLONINE HYDROCHLORIDE

DYPHYLLINE

ECAMSULE TRIETHANOLAMINE

ECONAZOLE NITRATE

EDETATE DISODIUM

EDITOL

EDOXUDINE

EMETINE

ENALAPRIL MALEATE

ENALAPRILAT

ENOXACIN

ENROFLOXACIN

ENTACAPONE

EPHEDRINE (1R,2S) HYDROCHLORIDE

EPINEPHRINE BITARTRATE

EPRINOMECTIN

ERGOCALCIFEROL

ERGONOVINE MALEATE

ERYTHROMYCIN

ERYTHROMYCIN ESTOLATE

ERYTHROMYCIN ETHYLSUCCINATE

ESCITALOPRAM OXALATE

ESOMEPRAZOLE POTASSIUM

(Continued) 
Table 1. List of compounds from the PHARMAKON 1600 library used in this screen. (continued)

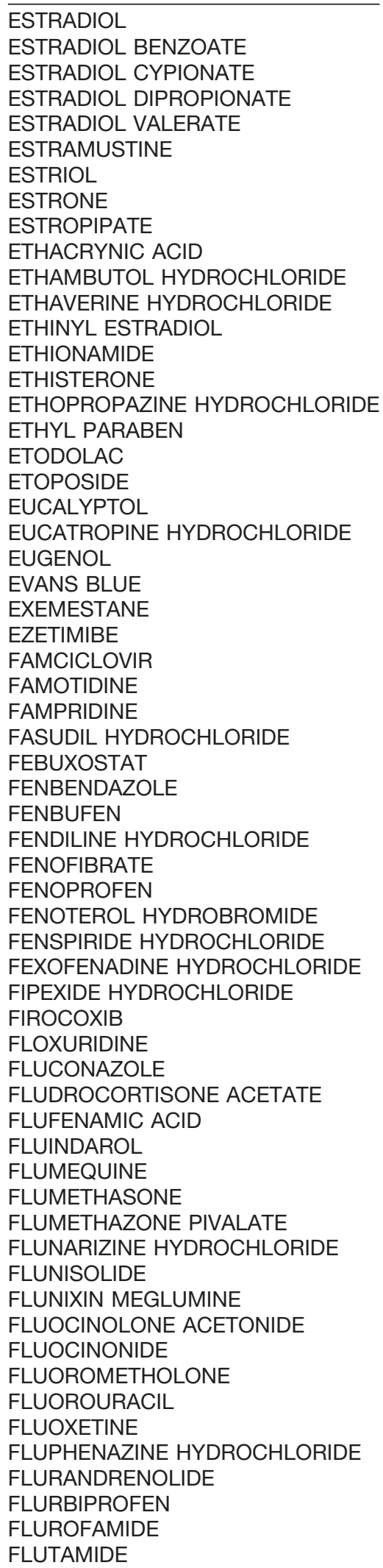

Table 1. List of compounds from the PHARMAKON 1600 library used in this screen. (continued)

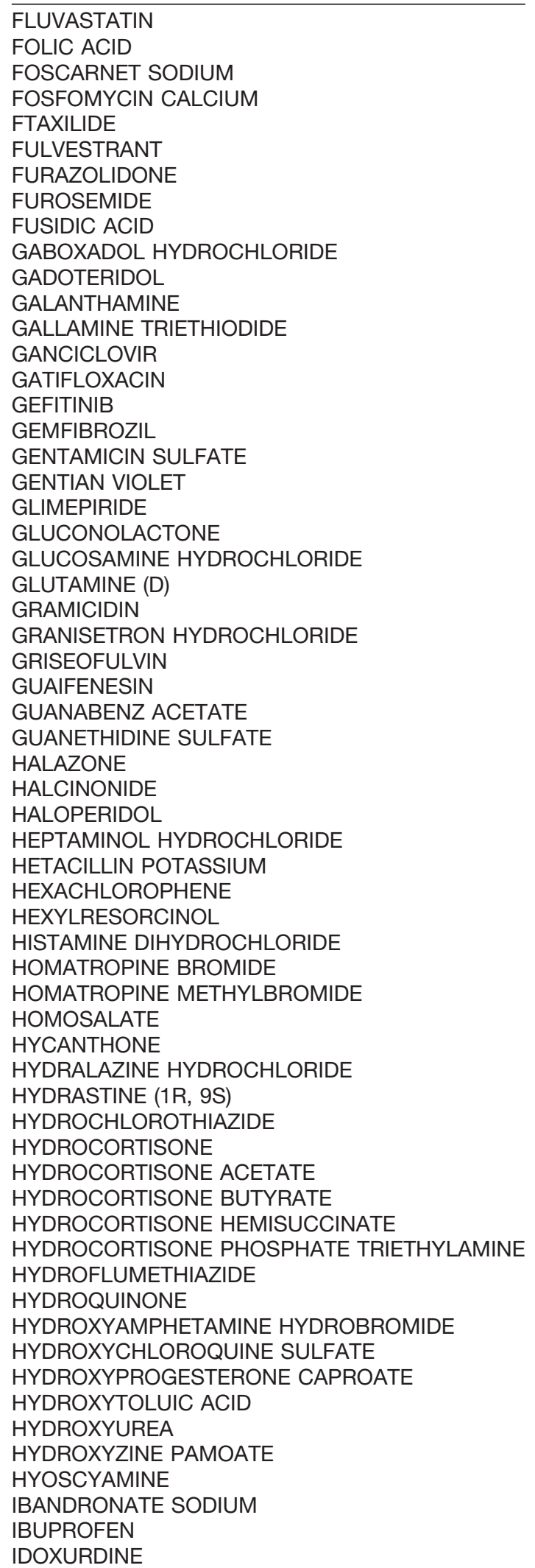


Table 1. List of compounds from the PHARMAKON 1600 library used in this screen. (continued)

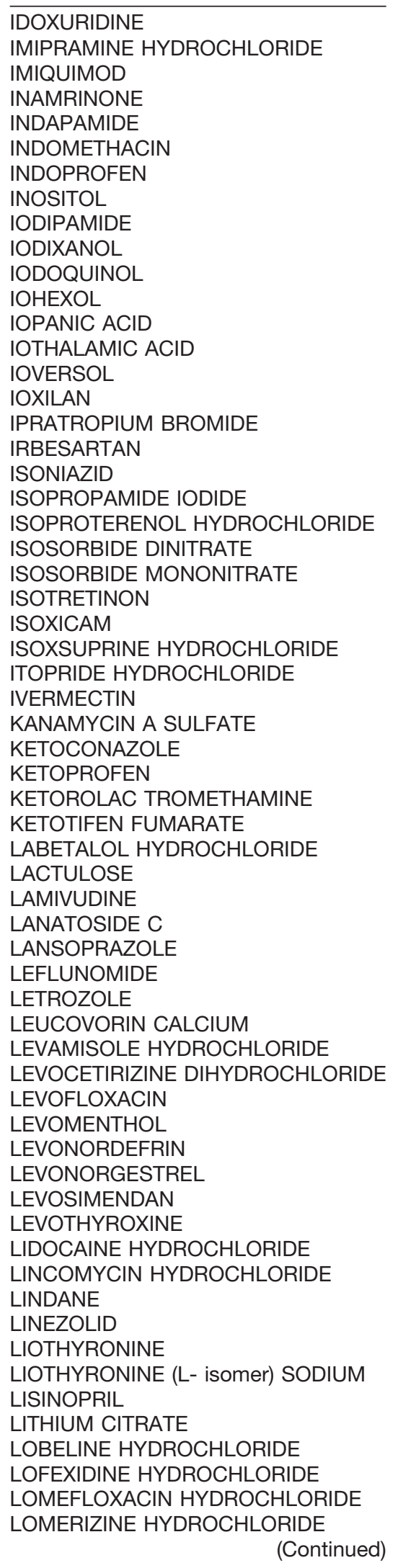

Table 1. List of compounds from the PHARMAKON 1600 library used in this screen. (continued)

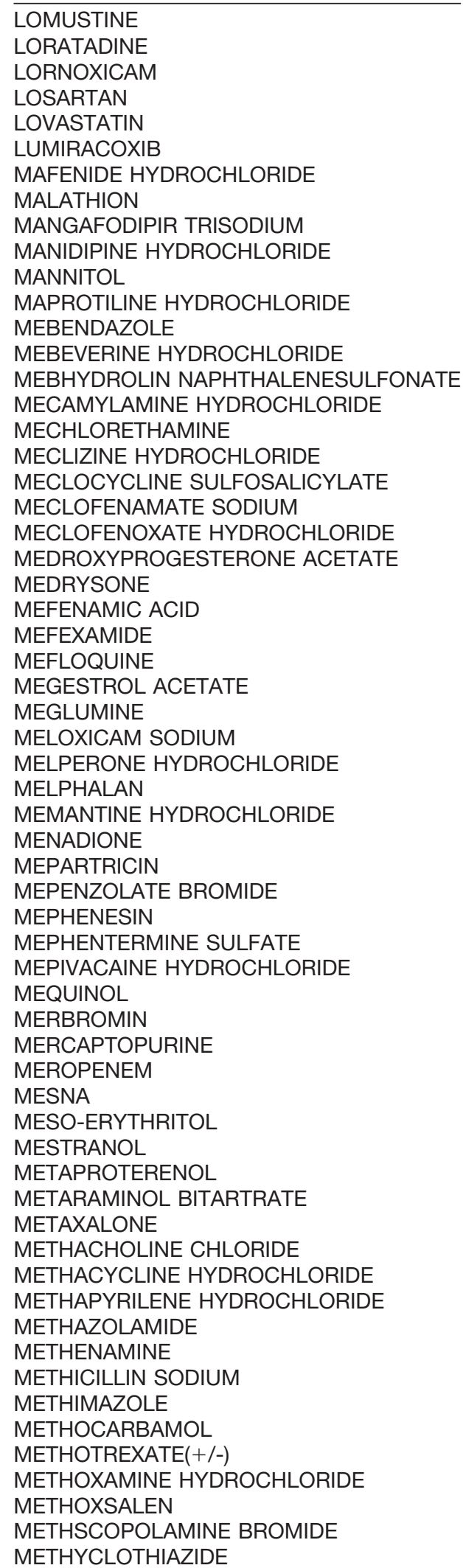

(Continued) 
Table 1. List of compounds from the PHARMAKON 1600 library used in this screen. (continued)

METHYLBENZETHONIUM CHLORIDE

METHYLDOPA

METHYLERGONOVINE MALEATE

METHYLPREDNISOLONE

METHYLPREDNISOLONE SODIUM SUCCINATE

METHYLTHIOURACIL

METOCLOPRAMIDE HYDROCHLORIDE

METOPROLOL TARTRATE

METRONIDAZOLE

MEXILETINE HYDROCHLORIDE

MICONAZOLE NITRATE

MIDODRINE HYDROCHLORIDE

MIGLITOL

MILNACIPRAN HYDROCHLORIDE

MINAPRINE HYDROCHLORIDE

MINOCYCLINE HYDROCHLORIDE

MINOXIDIL

MITOMYCIN C

MITOTANE

MITOXANTRONE HYDROCHLORIDE

MOLSIDOMINE

MONENSIN SODIUM (monensin A is shown)

MONOBENZONE

MORANTEL CITRATE

MOXALACTAM DISODIUM

MOXIFLOXACIN HYDROCHLORIDE

MYCOPHENOLATE MOFETIL

MYCOPHENOLIC ACID

NABUMETONE

NADIDE

NADOLOL

NAFCILLIN SODIUM

NAFRONYL OXALATE

NALBUPHINE HYDROCHLORIDE

NALIDIXIC ACID

NALOXONE HYDROCHLORIDE

NALTREXONE HYDROCHLORIDE

NAPHAZOLINE HYDROCHLORIDE

NAPROXEN $(+)$

NAPROXOL

NATEGLINIDE

NEFAZODONE HYDROCHLORIDE

NEFOPAM

NELARABIN

NEOMYCIN SULFATE

NEOSTIGMINE BROMIDE

NEVIRAPINE

NIACIN

NICARDIPINE HYDROCHLORIDE

NICERGOLINE

NICLOSAMIDE

NICOTINYL ALCOHOL TARTRATE

NIFEDIPINE

NIFURSOL

NILUTAMIDE

NIMODIPINE

NITAZOXANIDE

NITRENDIPINE

NITROFURANTOIN

NITROFURAZONE

NITROMIDE

NOCODAZOLE
Table 1. List of compounds from the PHARMAKON 1600 library used in this screen. (continued)

NOMIFENSINE MALEATE

NOREPINEPHRINE

NORETHINDRONE

NORETHINDRONE ACETATE

NORETHYNODREL

NORFLOXACIN

NORGESTREL

NORTRIPTYLINE

NOSCAPINE HYDROCHLORIDE

NOVOBIOCIN SODIUM

NYLIDRIN HYDROCHLORIDE

NYSTATIN

OCTOPAMINE HYDROCHLORIDE

OFLOXACIN

OLMESARTAN

OLMESARTAN MEDOXOMIL

OLSALAZINE SODIUM

OLSELTAMIVIR PHOSPHATE

OMEGA-3-ACID ESTERS (EPA shown)

ONDANSETRON

ORLISTAT

ORPHENADRINE CITRATE

OUABAIN

OXACILLIN SODIUM

OXALIPLATIN

OXCARBAZEPINE

OXETHAZAINE

OXIBENDAZOLE

OXIDOPAMINE HYDROCHLORIDE

OXOLINIC ACID

OXYBENZONE

OXYMETAZOLINE HYDROCHLORIDE

OXYPHENBUTAZONE

OXYPHENCYCLIMINE HYDROCHLORIDE

OXYQUINOLINE HEMISULFATE

OXYTETRACYCLINE

PACLITAXEL

PALIPERIDONE

PAPAVERINE HYDROCHLORIDE

PARACHLOROPHENOL

PARAROSANILINE PAMOATE

PARGYLINE HYDROCHLORIDE

PAROMOMYCIN SULFATE

PAROXETINE HYDROCHLORIDE

PEMETREXED

PENCICLOVIR

PENICILLAMINE

PENICILLIN G POTASSIUM

PENICILLIN V POTASSIUM

PENTOLINIUM TARTRATE

PENTOXIFYLLINE

PERGOLIDE MESYLATE

PERHEXILINE MALEATE

PERICIAZINE

PERINDOPRIL ERBUMINE

PERPHENAZINE

PHENACEMIDE

PHENAZOPYRIDINE HYDROCHLORIDE

PHENELZINE SULFATE

PHENINDIONE

PHENIRAMINE MALEATE

(Continued) 
Table 1. List of compounds from the PHARMAKON 1600 library used in this screen. (continued)

PHENOLPHTHALEIN

PHENTOLAMINE HYDROCHLORIDE

PHENYL AMINOSALICYLATE

PHENYLBUTAZONE

PHENYLEPHRINE HYDROCHLORIDE

PHENYLMERCURIC ACETATE

PHENYLPROPANOLAMINE HYDROCHLORIDE

PHENYTOIN SODIUM

PHTHALYLSULFATHIAZOLE

PHYSOSTIGMINE SALICYLATE

PILOCARPINE NITRATE

PIMOZIDE

PINDOLOL

PIOGLITAZONE HYDROCHLORIDE

PIPERACETAZINE

PIPERACILLIN SODIUM

PIPERAZINE

PIPERIDOLATE HYDROCHLORIDE

PIPERINE

PIPOBROMAN

PIRACETAM

PIRENPERONE

PIRENZEPINE HYDROCHLORIDE

PIROCTONE OLAMINE

PIROXICAM

PITAVASTATIN CALCIUM

PIZOTYLINE MALATE

POLYMYXIN B SULFATE

POTASSIUM $p$-AMINOBENZOATE

PRAMIPEXOLE DIHYDROCHLORIDE

PRAMOXINE HYDROCHLORIDE

PRASUGREL

PRAZIQUANTEL

PRAZOSIN HYDROCHLORIDE

PREDNICARBATE

PREDNISOLONE

PREDNISOLONE ACETATE

PREDNISONE

PRILOCAINE HYDROCHLORIDE

PRIMAQUINE DIPHOSPHATE

PRIMIDONE

PROADIFEN HYDROCHLORIDE

PROBENECID

PROBUCOL

PROCAINAMIDE HYDROCHLORIDE

PROCAINE HYDROCHLORIDE

PROCARBAZINE HYDROCHLORIDE

PROCHLORPERAZINE EDISYLATE

PROCYCLIDINE HYDROCHLORIDE

PROGESTERONE

PROGLUMIDE

PROMAZINE HYDROCHLORIDE

PROMETHAZINE HYDROCHLORIDE

PRONETALOL HYDROCHLORIDE

PROPAFENONE HYDROCHLORIDE

PROPANTHELINE BROMIDE

PROPIOLACTONE

PROPOFOL

PROPYLTHIOURACIL

PSEUDOEPHEDRINE HYDROCHLORIDE

(Continued)
Table 1. List of compounds from the PHARMAKON 1600 library used in this screen. (continued)

PUROMYCIN HYDROCHLORIDE

PYRANTEL PAMOATE

PYRAZINAMIDE

PYRETHRINS

PYRIDOSTIGMINE BROMIDE

PYRILAMINE MALEATE

PYRIMETHAMINE

PYRITHIONE ZINC

PYRONARIDINE TETRAPHOSPHATE

PYRVINIUM PAMOATE

QUETIAPINE

QUINACRINE HYDROCHLORIDE

QUINAPRIL HYDROCHLORIDE

QUINESTROL

QUINETHAZONE

QUINIDINE GLUCONATE

QUININE SULFATE

QUIPAZINE MALEATE

RACEPHEDRINE HYDROCHLORIDE

RACTOPAMINE HYDROCHLORIDE

RAMIPRIL

RAMOPLANIN [A2 shown; 2mm]

RANITIDINE

RASAGILINE

RESERPINE

RESORCINOL

RESORCINOL MONOACETATE

RETAPAMULIN

RETINOL

RETINYL PALMITATE

RIBAVIRIN

RIFAMPIN

RITANSERIN

RITODRINE HYDROCHLORIDE

RITONAVIR

RIZATRIPTAN BENZOATE

ROFECOXIB

RONIDAZOLE

ROPINIROLE

ROSIGLITAZONE

ROSUVASTATIN CALCIUM

ROXARSONE

ROXATIDINE ACETATE HYDROCHLORIDE

ROXITHROMYCIN

RUFLOXACIN HYDROCHLORIDE

SACCHARIN

SALICIN

SALICYL ALCOHOL

SALICYLAMIDE

SALICYLANILIDE

SALINOMYCIN, SODIUM

SALSALATE

SANGUINARINE SULFATE

SCOPOLAMINE HYDROBROMIDE

SELAMECTIN

SEMUSTINE

SENNOSIDE A

SERATRODAST

SERTRALINE HYDROCHLORIDE

SEVOFLURANE

SIBUTRAMINE HYDROCHLORIDE

(Continued) 
Table 1. List of compounds from the PHARMAKON 1600 library used in this screen. (continued)

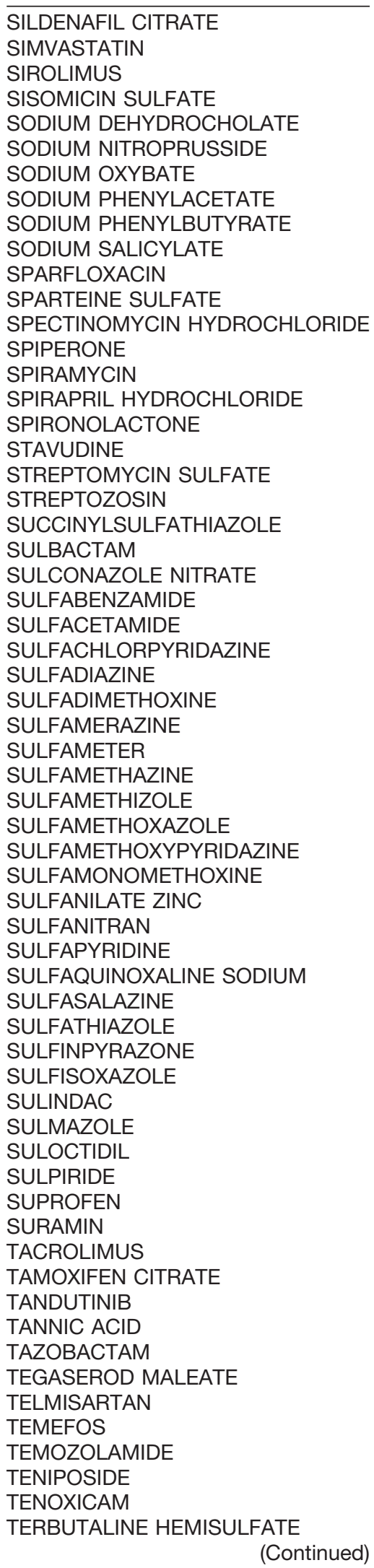

Table 1. List of compounds from the PHARMAKON 1600 library used in this screen. (continued)

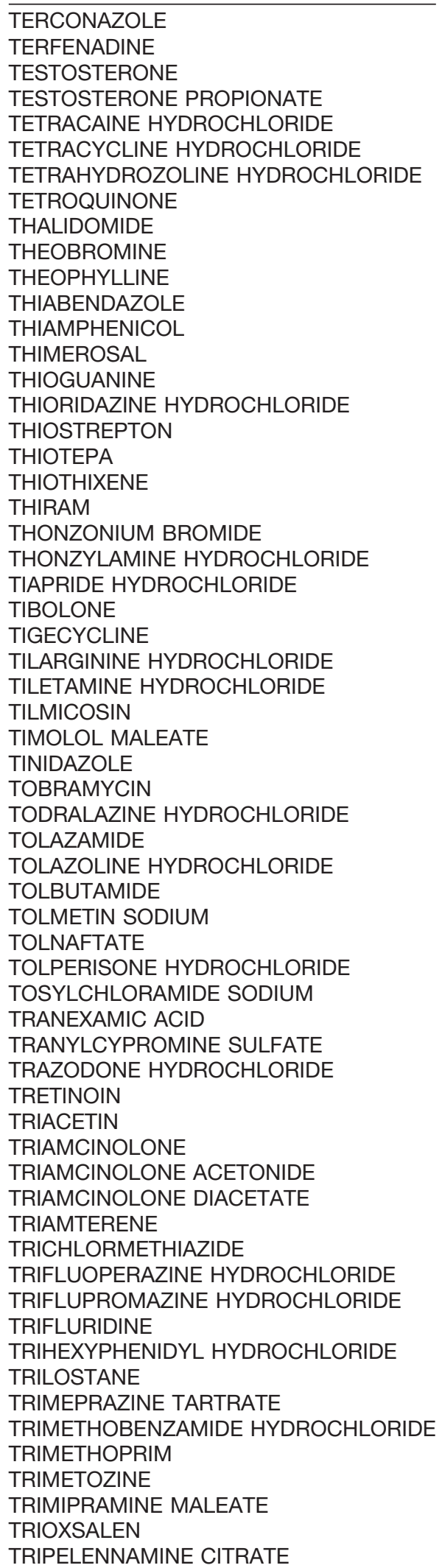


Table 1. List of compounds from the PHARMAKON 1600 library used in this screen. (continued)

\begin{tabular}{l}
\hline TRIPROLIDINE HYDROCHLORIDE \\
TRISODIUM ETHYLENEDIAMINE TETRACETATE \\
TROLEANDOMYCIN \\
TROPICAMIDE \\
TROPISETRON HYDROCHLORIDE \\
TRYPTOPHAN \\
TUAMINOHEPTANE SULFATE \\
TUBOCURARINE CHLORIDE \\
TYROTHRICIN \\
URACIL \\
URAPIDIL HYDROCHLORIDE \\
UREA \\
URETHANE \\
URSODIOL \\
VALDECOXIB \\
VALGANCICLOVIR HYDROCHLORIDE \\
VALPROATE SODIUM \\
VALSARTAN \\
VANCOMYCIN HYDROCHLORIDE \\
VENLAFAXINE \\
VIDARABINE \\
VINBLASTINE SULFATE \\
VINORELBINE \\
VINPOCETINE \\
VIOMYCIN SULFATE \\
VORICONAZOLE \\
VORINOSTAT \\
WARFARIN \\
XYLAZINE \\
XYLOMETAZOLINE HYDROCHLORIDE \\
YOHIMBINE HYDROCHLORIDE \\
ZALCITABINE \\
ZAPRINAST \\
ZIDOVUDINE [AZT] \\
ZIPRASIDONE MESYLATE \\
ZOMEPIRAC SODIUM \\
ZOPICLONE
\end{tabular}

were obtained from mutants bathed in embryo media, as described above; a second locomotion plot was then obtained following a solution change to a test compound and an equilibration period of 15-30 min. Criteria for a positive hit designation were as follows: (1) a decrease in mean velocity of $\geq 44 \%$ (e.g., a value based on the trialto-trial variability measured in control tracking studies; Fig. 1c); and (2) a reduction to Stage 0 or Stage I seizure behavior in the locomotion plot for at least $50 \%$ of the test fish. Each test compound classified as a "positive hit" in the locomotion assay was confirmed, under direct visualization on a stereomicroscope, as the fish being alive based on movement in response to external stimulation and a visible heartbeat following a 60 min drug exposure. Toxicity (or mortality) was defined as no visible heartbeat or movement in response to external stimulation in at least $50 \%$ of the test fish. Hyperexcitability was defined as a compound causing a $\geq 44 \%$ increase in swim velocity and/or Stage III seizure activity in at least $50 \%$ of the test fish. Hits identified in the primary locomotion screen were selected from the PHARMAKON 1600 library and rescreened using the method described above. Select com- pound stocks that were successful in two primary locomotion assays, and were not classified as toxic in two independent clutches of zebrafish, were then purchased separately from Sigma-Aldrich for further testing. Drug concentrations between 0.5 and $1 \mathrm{~mm}$ were used for electrophysiology assays to account for more limited diffusion in agar-embedded larvae.

\section{Data analysis}

Data are presented as the mean and SEM, unless stated otherwise. Pairwise statistical significance was determined with a Student's two-tailed unpaired $t$ test, ANOVA, or Mann-Whitney rank sum test, as appropriate, unless stated otherwise. Results were considered significant at $p$ $<0.05$, unless otherwise indicated.

\section{Results}

\section{A first-stage behavioral screen for antiepileptic activity}

Locomotion tracking is a reliable and rapid strategy with which to monitor behavioral seizures in freely swimming larval zebrafish (Baraban et al., 2005, 2013; Winter et al., 2008). In these locomotion plots, high-velocity movements of $\geq 20 \mathrm{~mm} / \mathrm{s}$ correspond to paroxysmal wholebody convulsions, referred to as Stage III, and are consistently observed in unprovoked scn1Lab mutant larvae but not in age-matched wild-type siblings. Using automated locomotion tracking, we performed a phenotype-based screen to identify compounds that significantly reduce mutant swim behavior to levels associated with Stage 0 or Stage I (e.g., activity equivalent to that seen in normal untreated WT zebrafish). In a 96-well format, we tracked mutant swim activity at baseline, and then again after addition of a test compound (100 $\mu \mathrm{M})$; each compound was tested on six individual mutant larvae (Fig. 1a), and larvae were sorted based on pigmentation differences (Fig. 1b). Mutant swim activity between two consecutive recording epochs in embryo media is tracked on every plate as an internal control. A box plot showing the change in swim velocity in untreated mutants is shown in Figure 1c $(n=112)$ and defined as the control. Based on an SD of 21.8 for these data, we set the detection threshold as any compound that inhibits movement (measured as a change in mean velocity) by $>2$ SDs (or $\geq 44 \%$ ). This approach was previously validated using standard antiepileptic drugs in this model (Baraban et al., 2013). Next, we screened a repurposed library in which all compounds have reached the clinical evaluation stage (PHARMAKON 1600 Collection; http://www.msdiscovery.com/pharma.html). Among the 1012 compounds screened (Fig. 1d) only 20 (or 1.97\%) were found to significantly inhibit spontaneous seizure behavior in scn1Lab mutants. All 20 compounds were subsequently retested in a separate clutch of scn1Lab mutants at a concentration of $100 \mu \mathrm{M}$ (Fig. 2a, trial 2; $N=6$ fish/ compound). A total of 154 compounds were classified as "toxic" (Table 2); 55 compounds were classified as "hyperexcitable" (Table 3). Representative locomotion tracking raw data plots for gemfibrozil, a toxic nonsteroid 
a

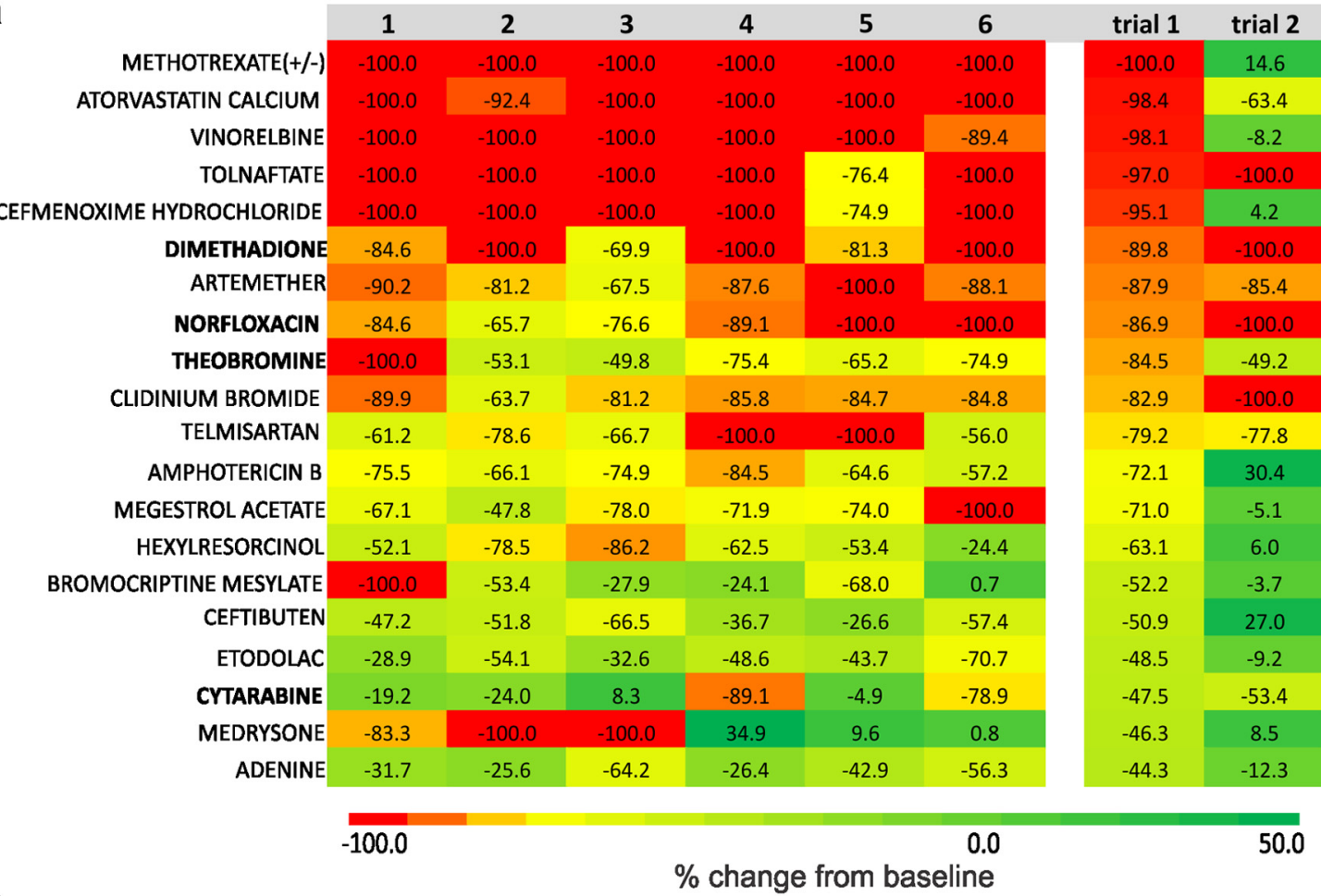

b
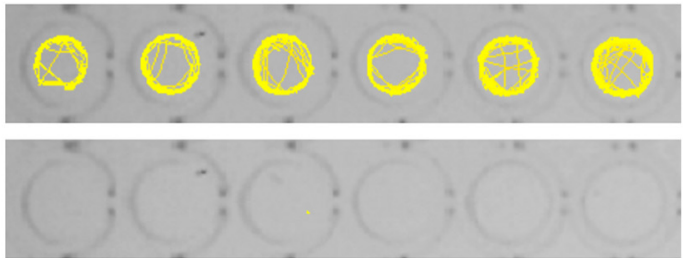

gemfibrozil
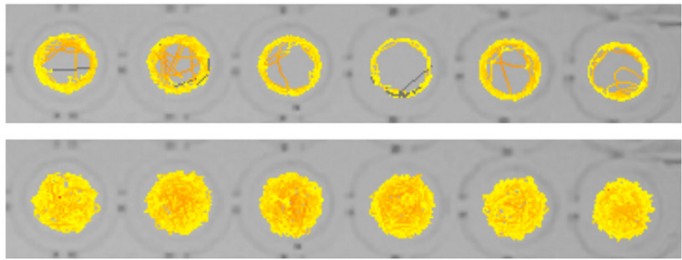

mepivacaine

Figure 2. Positive hits identified in the locomotion assay. a, Heat map showing the results of individual zebrafish trials (1-6) for compounds tested at a concentration of $100 \mu \mathrm{m}$ in the locomotion-tracking assay. Raw data values for individual fish are shown within the color-coded boxes for one sample trial. Mean velocity data are shown at right for "trial 1" and "trial 2"; six fish per trial. Note: only drugs highlighted in bold type were classified as positive nontoxic hits in two independent trials and moved on to further testing. $\boldsymbol{b}$, Representative raw locomotion data plots for six individual scn1Lab mutant larvae at baseline (top) and following the addition of a compound resulting in fatality (bottom, gemfibrozil) or hyperactivity (bottom, mepivacaine). Movement is color coded, with lowvelocity movements shown in yellow, and high velocity movements shown in red.

nuclear receptor ligand, and mepivacaine, a hyperexcitable proconvulsant anesthetic, are shown in Figure $2 b$.

\section{A second-stage electrophysiology assay for antiepileptic activity}

Extracellular recording electrodes are a reliable, reproducible, and sensitive approach to monitor electroencephalographic activity in agar-immobilized larval zebrafish (Baraban et al., 2005; Baraban, 2013). Field electrodes offer high a signal-to-noise ratio and can be placed, using direct visualization in transparent larvae, into specific CNS structures (i.e., telencephalon or optic tectum). Using a local field electrode, we can efficiently monitor the occurrence of electrographic seizure events in the same zebrafish that were previously tested in the locomotion assay. Based on a positive nontoxic result in two independent locomotion assays, four drugs moved on to electrophysiology testing at concentrations between $500 \mu \mathrm{M}$ and $1 \mathrm{~mm}$ (Fig. 3). Consistent with a "falsepositive" classification, spontaneous epileptiform discharge activity was observed for three of these drugs: norfloxacin, theobromine, and cytarabine. Dimethadione, previously shown to inhibit spontaneous epileptiform discharges in thalamocortical slices at concentrations between 1 and $10 \mathrm{~mm}$ (Zhang et al., 1996), suppressed burst discharge activity in scn1Lab mutant larvae (Fig. 3a,b). To identify whether any of these four compounds exert nonspecific effects on behavior, they were also tested on freely swimming WT zebrafish larvae (5 dpf) at a concentration of $500 \mu \mathrm{m}$. Comparing the total distance moved during a 10 min recording epoch before, and after, the application of a test compound failed to reveal any significant changes in locomotor activity (Fig. 3c). 
Table 2: List of compounds exhibiting toxicity.

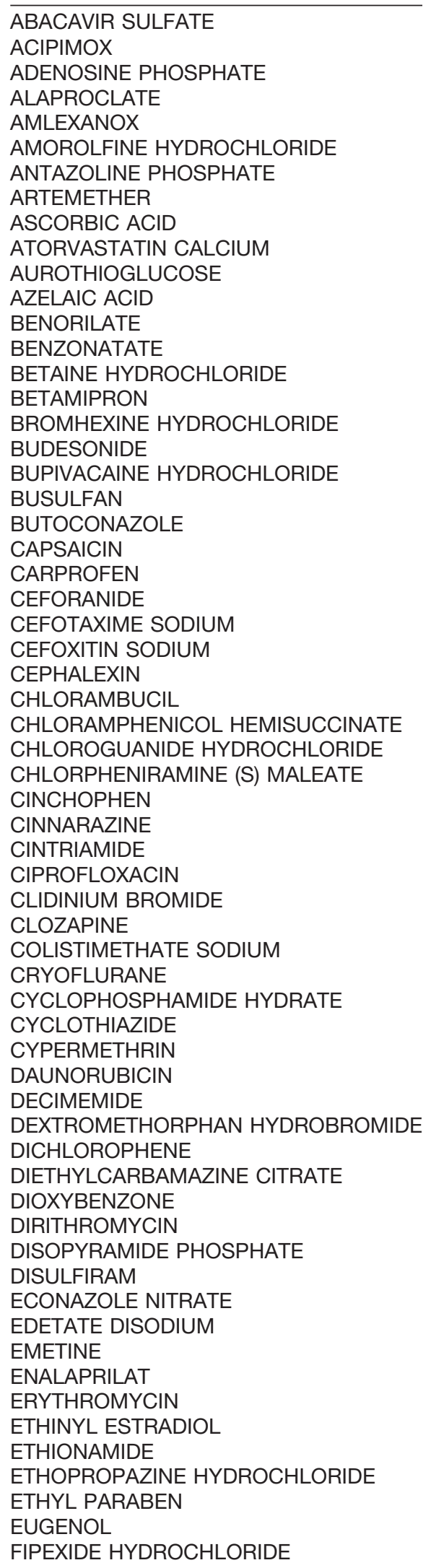

Table 2: List of compounds exhibiting toxicity.

FLUMETHASONE

FLUNISOLIDE

FLUVASTATIN

GEMFIBROZIL

GENTAMICIN SULFATE

GLUCONOLACTONE

HALAZONE

HALCINONIDE

HETACILLIN POTASSIUM

HEXACHLOROPHENE

HOMATROPINE METHYLBROMIDE

HYDRASTINE (1R, 9S)

HYDROXYAMPHETAMINE HYDROBROMIDE HYDROXYCHLOROQUINE SULFATE IODIXANOL

IOHEXOL

IRBESARTAN

LEVOSIMENDAN

LISINOPRIL

LOMERIZINE HYDROCHLORIDE

MANGAFODIPIR TRISODIUM

MECLOFENOXATE HYDROCHLORIDE

MESTRANOL

METHACHOLINE CHLORIDE

METHYLERGONOVINE MALEATE

METRONIDAZOLE

MIGLITOL

MONENSIN SODIUM (monensin A is shown) MONOBENZONE

MOXALACTAM DISODIUM

NADOLOL

NALBUPHINE HYDROCHLORIDE

NALTREXONE HYDROCHLORIDE

NAPHAZOLINE HYDROCHLORIDE

NAPROXEN $(+)$

NEOMYCIN SULFATE

NIFEDIPINE

NITAZOXANIDE

NITROMIDE

NORETHINDRONE

OLMESARTAN MEDOXOMIL

OXYMETAZOLINE HYDROCHLORIDE

PARACHLOROPHENOL

PAROMOMYCIN SULFATE

PERHEXILINE MALEATE

PHENTOLAMINE HYDROCHLORIDE

PHENYLBUTAZONE

PHENYLMERCURIC ACETATE

PHYSOSTIGMINE SALICYLATE

PIMOZIDE

PIPERACILLIN SODIUM

PIPERAZINE

PIRACETAM

PIRENZEPINE HYDROCHLORIDE

PIROCTONE OLAMINE

PITAVASTATIN CALCIUM

PRIMAQUINE DIPHOSPHATE

PROBENECID

PROCARBAZINE HYDROCHLORIDE

PROGLUMIDE

PROMETHAZINE HYDROCHLORIDE

PUROMYCIN HYDROCHLORIDE

(Continued) 
Table 2: List of compounds exhibiting toxicity.

\begin{tabular}{l}
\hline QUININE SULFATE \\
RETINYL PALMITATE \\
RIFAMPIN \\
RITONAVIR \\
ROFECOXIB \\
RUFLOXACIN HYDROCHLORIDE \\
SACCHARIN \\
SALICIN \\
SENNOSIDE A \\
STAVUDINE \\
STREPTOMYCIN SULFATE \\
SULFADIAZINE \\
SULINDAC \\
SULOCTIDIL \\
TANNIC ACID \\
TELMISARTAN \\
TENOXICAM \\
THEOPHYLLINE \\
TILETAMINE HYDROCHLORIDE \\
TILMICOSIN \\
TIMOLOL MALEATE \\
TOLBUTAMIDE \\
TOLNAFTATE \\
TRAZODONE HYDROCHLORIDE \\
TRETINOIN \\
TRIFLUPROMAZINE HYDROCHLORIDE \\
TROPISETRON HYDROCHLORIDE \\
VALDECOXIB \\
VORINOSTAT \\
ZALCITABINE \\
\hline
\end{tabular}

\section{Assessment of huperzine A and fenfluramine for antiepileptic activity}

Next, we tested two additional compounds that were not in our drug library, but have recently been described as potential antiepileptic treatments for DS. Huperzine A, a small-molecule alkaloid isolated from Chinese club moss with NMDA-type receptor blocking and anticholinesterase activity, has purported antiepileptic actions against NMDA- or soman-induced seizures (Tonduli et al., 2001; Coleman et al., 2008). In the locomotion assay, huperzine A failed to significantly alter scn1Lab seizure behavior at any concentration tested (Fig. $4 a, b$ ). In contrast, huperzine A was effective at $1 \mathrm{~mm}$ in the acute pentylenetetrazole (PTZ) assay (Fig. 4b). Fenfluramine is an amphetamine-like compound that has been reported to successfully reduce seizure occurrence in children with DS as a low-dose add-on therapy (Ceulemans et al., 2012). In the locomotion assay, fenfluramine significantly reduced mutant mean swim velocity at concentrations between 100 and $500 \mu \mathrm{M}$ (Fig. 4c,d); $1 \mathrm{~mm}$ fenfluramine was toxic in the scn1Lab and PTZ assays (Fig. 4d). The fenfluramine-treated scn1Lab mutant exhibited a suppression of spontaneous electrographic seizure discharge to levels similar to controls at $500 \mu \mathrm{M}$, but only a partial reduction in electrographic activity at $250 \mu \mathrm{M}$ (Fig. 4e).

\section{Discussion}

Zebrafish and humans share extensive genomic similarity. With regard to disease, $84 \%$ of genes known to be associated with disease states in humans have a zebrafish
Table 3: List of compounds exhibiting hyperexcitable or proconvulsant activity.

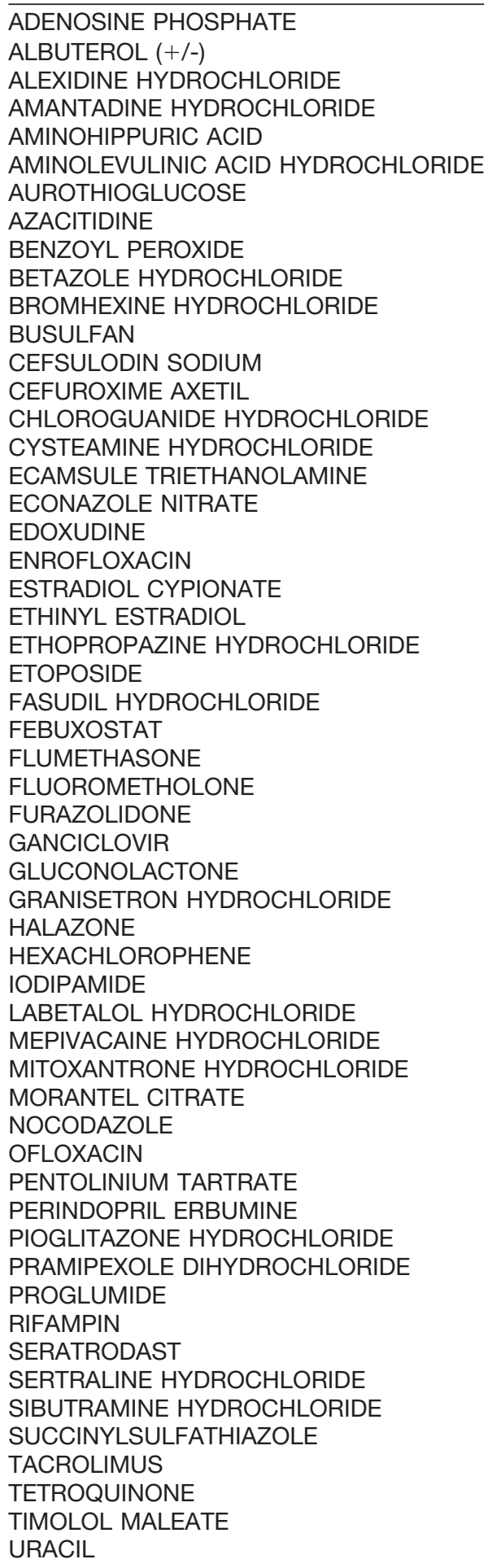

homolog (Howe et al., 2013). This genetic similarity and the characteristic of zebrafish larvae to exhibit quantifiable seizure behaviors or electrographic seizure discharge that is fundamentally similar to that recorded in humans (Jirsa et al., 2014) make this an ideal system for drug discovery. Behavioral assays customized for auto- 
a cytarabine

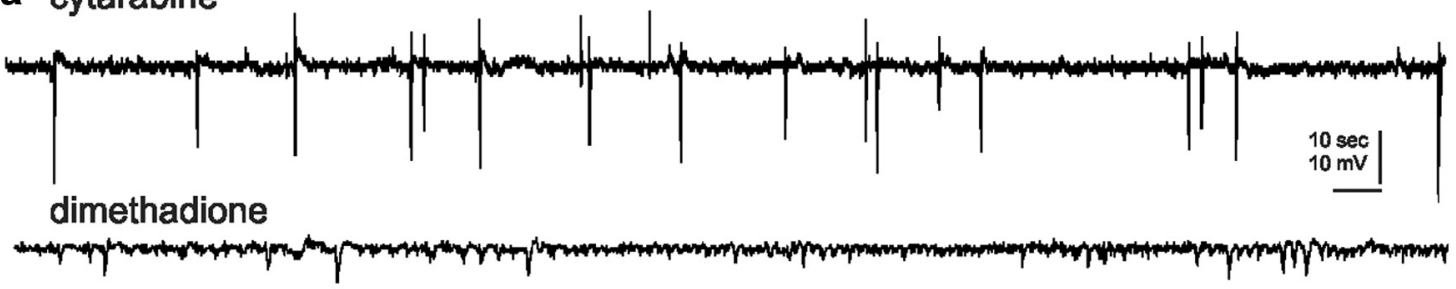

\section{theobromine}
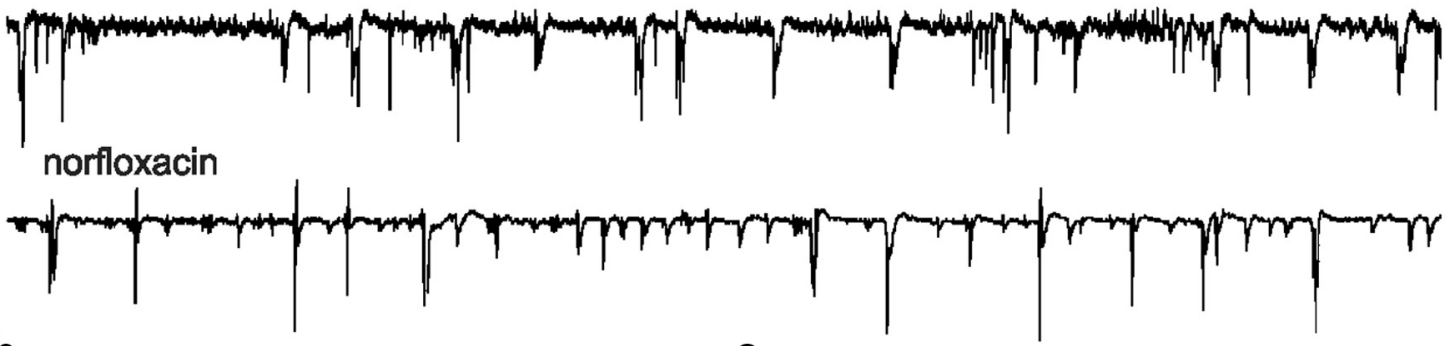

b

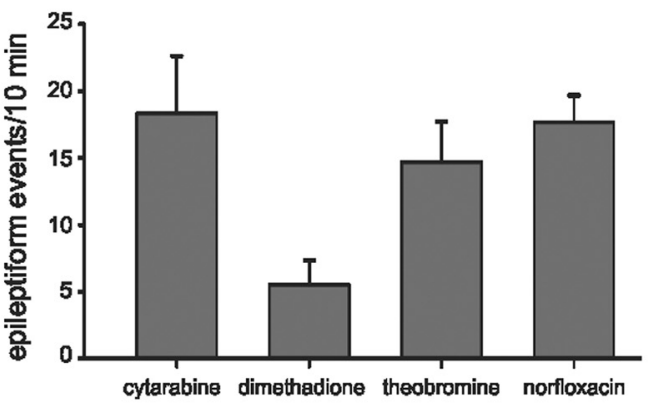

C

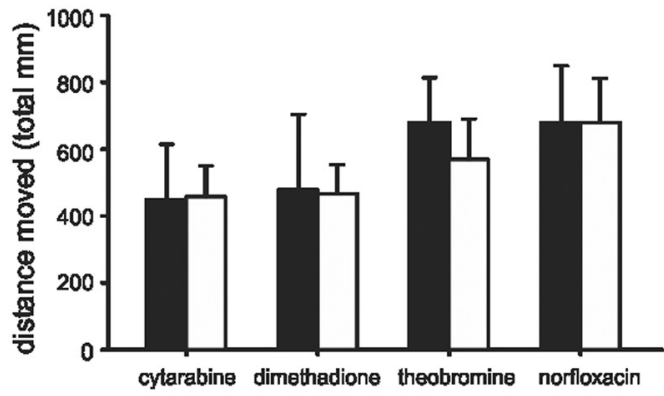

Figure 3. Electrophysiology assay to identify drugs that rescue the scn1Lab mutant epilepsy phenotype. a, Representative field electrode recording epochs (5 min in duration) are shown for the "positive" compounds identified in the locomotion assay. All recordings were obtained with an electrode placed in the forebrain of agar-immobilized scn1Lab larvae that was previously tested in the locomotion assay. A suppression of epileptiform electrographic discharge activity was noted in mutants exposed to dimethadione. $\boldsymbol{b}$, Bar plot showing the mean number of epileptiform events in a 10 min recording epoch for scn 1 Lab larvae exposed to cytarabine $(N=6)$, dimethadione $(N=6)$, theobromine $(N=6)$, and norfloxacin $(N=6)$. The mean \pm SEM is shown. The fish shown were tested in the locomotion assay first. $\boldsymbol{c}$, Bar plot showing the total distance traveled before (black bars) and after (white bars) exposure to a test compound; 10 min recording epoch and six fish per drug. The mean \pm SEM is shown.

mated evaluation of locomotion (Winter et al., 2008; Creton, 2009; Baxendale et al., 2012; Baraban et al., 2013; Raftery et al., 2014) make moderate-to-high-throughput phenotype-based drug screening in zebrafish possible. Using this approach and a zebrafish scn1 mutant (Baraban et al., 2013), we successfully identified antiepileptic compounds. Here we report results from screening $\sim 1000$ compounds from a repurposed drug library and present data that will be periodically updated on-line using this open-access publishing mechanism.

As a model system, the scn1Lab mutant zebrafish has many advantages. First, in contrast to transient and variable knockdown of gene expression using antisense morpholino oligonucleotides (Teng et al., 2010; Finckbeiner et al., 2011; Mahmood et al., 2013), scn1Lab mutants carry a stable and heritable amino acid substitution at position 1208 in the third domain of SCN1A that shares $76 \%$ homology with humans (Schoonheim et al., 2010; Baraban et al., 2013). Mutations in this channel are one of the primary genetic causes underlying DS (Claes et al., 2003; Escayg and Goldin, 2010; De Jonghe, 2011; Saitoh et al., 2012). As zebrafish possess two scn1 genes (Novak et al., 2006), homozygous mutants for scn1 Lab are comparable to the haploinsufficient clinical condition, and there is no variability from larvae to larvae, or clutch to clutch, with respect to gene inactivation, as is commonly observed with morpholino injections (Kok et al., 2015). Although crosses of heterozygotes produce only onequarter homozygous scn1Lab mutants per mating, there are virtually no limitations on maintaining a large colony of healthy, adult breeders for these types of large-scale screens. Second, it is possible to observe and monitor seizure-like behavior consisting of rapid movements and whole-body convulsions in freely swimming scn1 Lab mutants as early as $4 \mathrm{dpf}$ that persist for the life of the larvae ( $\sim 12 \mathrm{dpf})$. These behaviors are comparable to those observed with exposure to a common convulsant agent (PTZ) and classified as Stage III (Baraban et al., 2005). In 
a

baseline (scn1Lab)

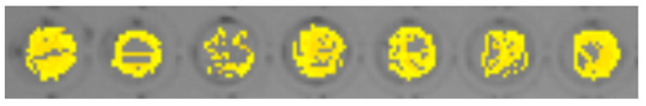

$1000 \mu \mathrm{M}$ huperzine $\mathrm{A}$

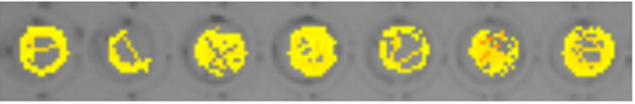

C

baseline (scn1Lab)

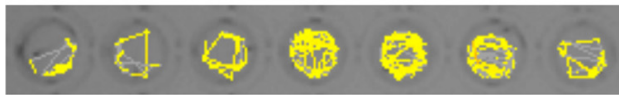

$500 \mu \mathrm{M}$ fenfluramine

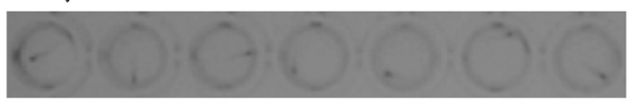

b

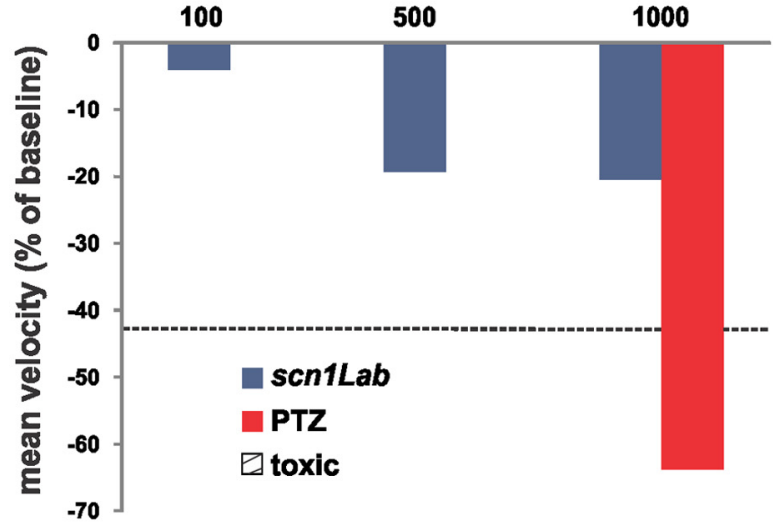

d

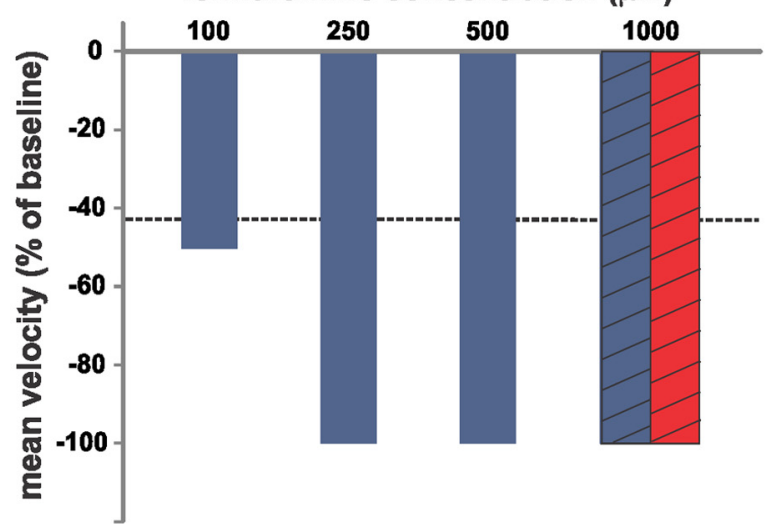

e

scn1Lab

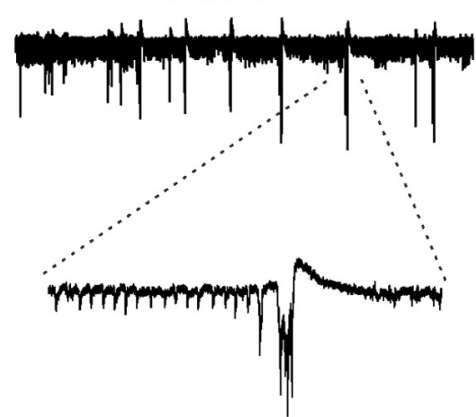

$250 \mu \mathrm{M}$ fenfluramine

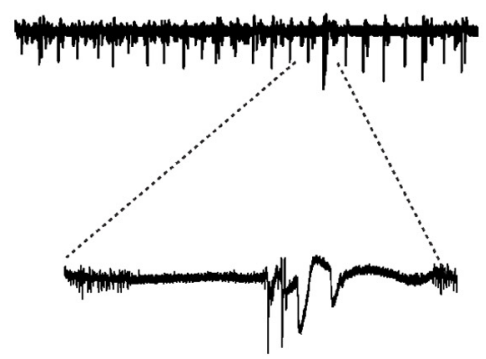

$500 \mu \mathrm{M}$ fenfluramine

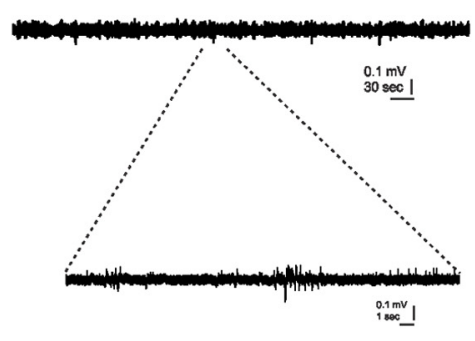

Figure 4. Evaluation of putative antiepileptic drugs in scn1Lab mutants. a, Locomotion tracking plots for scn1Lab zebrafish at baseline and following huperzine A administration. Total movement is shown for a 10 min recording epoch. $\boldsymbol{b}$, Plot showing the change in mean velocity for three different huperzine A concentrations (blue bars). Each bar is the mean change for six fish. The threshold for a positive hit is shown as a dashed line. WT fish exposed to PTZ and huperzine A are shown in red $(N=7)$. $\boldsymbol{c}, \boldsymbol{d}$, Same for fenfluramine. Note that $1 \mathrm{~mm}$ fenfluramine was toxic, as indicated. e, Representative field recordings from scn 1 Lab mutant larvae at 5 dpf. Electrographic activity is shown for a 5 min recording epoch (top traces); high-resolution traces are shown below, as indicated. Note that abnormal burst discharge activity persists in scn1Lab mutants exposed to $250 \mu \mathrm{m}$ fenfluramine. The fish shown were tested in the locomotion assay first.

addition, clear evidence for epileptiform discharge generated in the CNS of immobilized scn1Lab mutant larvae has been obtained at ages between 4 and $8 \mathrm{dpf}$ (Baraban et al., 2013). Both zebrafish measures of seizure activity are sensitive to inhibition by AEDs commonly prescribed to children with DS (e.g., valproate, benzodiazepines, and stiripentol), but are resistant to many antiepileptic compounds (e.g., phenytoin, carbamazepine, ethosuximide, decimemide, tiletamine, primidone, phenacemide, and vigabatrin). Pharmacoresistance is defined as the inability to control seizure activity with at least two different AEDs (Berg, 2009), and, with demonstrated resistance to eight 
AEDs, our model clearly fits this definition. This level of model validation has not been possible with morpholinos probably owing to the high degree of variability, or offtarget effects, associated with this technique (Kok et al., 2015).

Our screening results highlight the stringency of our approach with a positive hit rate of only $1.97 \%$ on the first-stage locomotion assay, and successful identification of 1 compound (of 1012 compounds) with known antiepileptic activity (i.e., dimethadione, a T-type channel antagonist). In additional testing, we confirmed an antiepileptic action for fenfluramine (serotonin uptake inhibitor). Similar to ethosuximide, a reduction in regenerative burst discharges associated with neuronal T-type calcium currents could be the underlying mechanism for dimethadione in DS mutants; however, it is worth noting that T-type channel blockers ethosuximide and flunarizine were not similarly effective (Baraban et al. 2013; this article), and that dimethadione can cause arrhythmia owing to blockade of cardiac human ether-a-go-go-related gene potassium channels (Azarbayjani and Danielsson, 2002; Danielsson et al., 2007). Modulation of serotonin [5-hydroxytryptamine (5-HT)] signaling by blocking uptake or increasing release from neurons by acting as substrates for 5-HT transporter (sertraline) proteins (Fuller et al., 1988; Gobbi and Mennini, 1999; Baumann et al., 2000; Rothman et al., 2010) may be the mechanism of action for fenfluramine in patients with DS, though a detailed analysis of precisely how fenfluramine modulates excitability via this signaling pathway has not been performed. Nonetheless, both drugs probably exert a direct effect on network excitability (at neuronal or synaptic levels, respectively) to suppress electrographic discharge and the associated high-velocity seizure behavior seen in scn1Lab mutants, and may be potential targets for clinical use. In contrast, three other drugs identified in the primary locomotion assay were not effective in suppressing electrical events and were designated as false positives. This is not altogether surprising given that the xanthine alkaloid (theobromine), chemotherapeutic (cytarabine), and antibiotic (norfloxacin) mechanisms for these compounds would not be consistent with seizure inhibition. Moreover, the variability inherent in behavioral experiments performed on different zebrafish larvae, in different microplates, and on different days may contribute to these false-positive designations in locomotion assays, and is evident in the range of mean velocity values seen during tracking episodes from control studies (Fig. 1C) or in the failure of many of the initial 20 lead compounds to be confirmed on subsequent retesting (see Fig. 2a). This is a limitation of locomotion-based screening assays and is another reason why a secondary electrophysiology assay on the same zebrafish is a critical advantage of our approach.

An additional advantage of in vivo screening with zebrafish larvae is the simultaneous identification of compounds resulting in toxicity. Zebrafish-based anticonvulsant drug-screening assays based primarily on in situ hybridization detection of early gene expression at $2 \mathrm{dpf}$ (Baxendale et al., 2012) do not routinely monitor sponta- neous swim behavior, heart rate, or response to external stimuli. Lacking these real-time measures of toxicity, compounds observed to induce fatality in a freely swimming scn1Lab-based behavioral assay (e.g., gemfibrozil, suloctidil, pimozide, or dioxybenzone) were mistakenly classified as seizure-suppressing compounds in the PTZbased c-Fos in situ hybridization assay. Indeed, $41 \%$ of the "anticonvulsant" compounds positively identified at 2 dpf in Baxendale et al. (2012) were toxic, proconvulsant, or simply not effective in scn1Lab mutant assays at 5-6 dpf. Similarly, it is critical to monitor blood flow and heart activity even in the agar-immobilized electrophysiology assay as compounds effective in suppressing electrical activity can also be toxic. These discrepancies highlight the potential problems associated with zebrafish drugscreening strategies that do not encompass multiple readouts and suggest the need for a note of caution when comparing screening results from different laboratory groups. While any lead compound identified in a zebrafish-based screening assay will, ultimately, need to be independently replicated and/or validated in additional mammalian model systems, the ability to rapidly identify such compounds, while simultaneously identifying potential negative side effects, makes genetically modified zebrafish a unique resource for drug discovery in an age of personalized medicine.

\section{References}

Ali S, Champagne DL, Spaink HP, Richardson MK (2011) Zebrafish embryos and larvae: a new generation of disease models and drug screens. Birth Defects Res C Embryo Today 93:115-133. CrossRef Medline

Azarbayjani F, Danielsson BR (2002) Embryonic arrhythmia by inhibition of HERG channels: a common hypoxia-related teratogenic mechanism for antiepileptic drugs? Epilepsia 43:457-468. Medline

Baraban SC, Dinday MT, Hortopan GA (2013) Drug screening in Scn1a zebrafish mutant identifies clemizole as a potential Dravet syndrome treatment. Nat Commun 4:2410. CrossRef Medline

Baraban SC, Taylor MR, Castro PA, Baier H (2005) Pentylenetetrazole induced changes in zebrafish behavior, neural activity and c-fos expression. Neuroscience 131:759-768. CrossRef Medline

Baumann MH, Ayestas MA, Dersch CM, Brockington A, Rice KC, Rothman RB (2000) Effects of phentermine and fenfluramine on extracellular dopamine and serotonin in rat nucleus accumbens: therapeutic implications. Synapse 36:102-113. CrossRef Medline

Baxendale S, Holdsworth CJ, Meza Santoscoy PL, Harrison MR, Fox J, Parkin CA, Ingham PW, Cunliffe VT (2012) Identification of compounds with anti-convulsant properties in a zebrafish model of epileptic seizures. Dis Model Mech 5:773-784. CrossRef Medline

Berg AT (2009) Identification of pharmacoresistant epilepsy. Neurol Clin 27:1003-1013. CrossRef Medline

Bialer M, Johannessen SI, Levy RH, Perucca E, Tomson T, White HS (2015) Progress report on new antiepileptic drugs: a summary of the Twelfth Eilat Conference (EILAT XII). Epilepsy Res 111:85-141. CrossRef Medline

Boel M, Casaer P (1996) Add-on therapy of fenfluramine in intractable self-induced epilepsy. Neuropediatrics 27:171-173. CrossRef Medline

Caraballo RH, Cersosimo RO, Sakr D, Cresta A, Escobal N, Fejerman N (2005) Ketogenic diet in patients with Dravet syndrome. Epilepsia 46:1539-1544. CrossRef Medline

Ceulemans B, Boel M, Leyssens K, Van Rossem C, Neels P, Jorens PG, Lagae $L$ (2012) Successful use of fenfluramine as an add-on treatment for Dravet syndrome. Epilepsia 53:1131-1139. CrossRef Medline 
Chiron C, Dulac O (2011) The pharmacologic treatment of Dravet syndrome. Epilepsia 52 Suppl 2:72-75. CrossRef Medline

Claes L, Ceulemans B, Audenaert D, Smets K, Löfgren A, Del-Favero J, Ala-Mello S, Basel-Vanagaite L, Plecko B, Raskin S, Thiry P, Wolf NI, Van Broeckhoven C, De Jonghe P (2003) De novo SCN1A mutations are a major cause of severe myoclonic epilepsy of infancy. Hum Mutat 21:615-621. CrossRef Medline

Coleman BR, Ratcliffe RH, Oguntayo SA, Shi X, Doctor BP, Gordon RK, Nambiar MP (2008) [+]-Huperzine A treatment protects against $\mathrm{N}$-methyl-D-aspartate-induced seizure/status epilepticus in rats. Chem Biol Interact 175:387-395. CrossRef Medline

Creton R (2009) Automated analysis of behavior in zebrafish larvae. Behav Brain Res 203:127-136. CrossRef Medline

Danielsson BR, Danielsson C, Nilsson MF (2007) Embryonic cardiac arrhythmia and generation of reactive oxygen species: common teratogenic mechanism for IKr blocking drugs. Reprod Toxicol 24:42-56. CrossRef Medline

De Jonghe P (2011) Molecular genetics of Dravet syndrome. Dev Med Child Neurol 53 Suppl 2:7-10. CrossRef Medline

Dravet C, Bureau M, Oguni H, Fukuyama Y, Cokar O (2005) Severe myoclonic epilepsy in infancy: Dravet syndrome. Adv Neurol 95: 71-102. Medline

Dressler A, Trimmel-Schwahofer P, Reithofer E, Mühlebner A, Gröppel G, Reiter-Fink E, Benninger F, Grassl R, Feucht M (2015) Efficacy and tolerability of the ketogenic diet in Dravet syndrome Comparison with various standard antiepileptic drug regimen. Epilepsy Res 109:81-89. CrossRef Medline

ebrary Inc. (2011) Guide for the care and use of laboratory animals. Washington, DC: National Academy Press.

Epi4K Consortium (2012) Epi4K: gene discovery in 4,000 genomes. Epilepsia 53:1457-1467. CrossRef

Escayg A, Goldin AL (2010) Sodium channel SCN1A and epilepsy: mutations and mechanisms. Epilepsia 51:1650-1658. CrossRef Medline

Finckbeiner S, Ko PJ, Carrington B, Sood R, Gross K, Dolnick B, Sufrin J, Liu $P$ (2011) Transient knockdown and overexpression reveal a developmental role for the zebrafish enosf $1 \mathrm{~b}$ gene. Cell Biosci 1:32. CrossRef Medline

Fuller RW, Snoddy HD, Robertson DW (1988) Mechanisms of effects of d-fenfluramine on brain serotonin metabolism in rats: uptake inhibition versus release. Pharmacol Biochem Behav 30:715-721. Medline

Gobbi M, Mennini T (1999) Release studies with rat brain cortical synaptosomes indicate that tramadol is a 5-hydroxytryptamine uptake blocker and not a 5-hydroxytryptamine releaser. Eur $\mathrm{J}$ Pharmacol 370:23-26. Medline

Howe K, Clark MD, Torroja CF, Torrance J, Berthelot C, Muffato M, Collins JE, Humphray S, McLaren K, Matthews L, McLaren S, Sealy I, Caccamo M, Churcher C, Scott C, Barrett JC, Koch R, Rauch GJ, White S, Chow W, et al (2013) The zebrafish reference genome sequence and its relationship to the human genome. Nature 496:498-503.

Jirsa VK, Stacey WC, Quilichini PP, Ivanov Al, Bernard C (2014) On the nature of seizure dynamics. Brain 137:2210-2230. CrossRef Medline

Ko HC, Gelb BD (2014) Concise review: drug discovery in the age of the induced pluripotent stem cell. Stem Cells Transl Med 3:500509. CrossRef Medline

Kok FO, Shin M, Ni CW, Gupta A, Grosse AS, van Impel A, Kirchmaier BC, Peterson-Maduro J, Kourkoulis G, Male I, DeSantis DF, Sheppard-Tindell S, Ebarasi L, Betsholtz C, Schulte-Merker S, Wolfe SA, Lawson ND (2015) Reverse genetic screening reveals poor correlation between morpholino-induced and mutant phenotypes in zebrafish. Dev Cell 32:97-108. CrossRef Medline

Leppert MF (1990) Gene mapping and other tools for discovery. Epilepsia 31 [Suppl 3]:S11-S18. Medline

Lotte J, Haberlandt E, Neubauer B, Staudt M, Kluger GJ (2012) Bromide in patients with SCN1A-mutations manifesting as Dravet syndrome. Neuropediatrics 43:17-21. CrossRef Medline

Lowson S, Gent JP, Goodchild CS (1990) Anticonvulsant properties of propofol and thiopentone: comparison using two tests in laboratory mice. $\mathrm{Br} \mathrm{J}$ Anaesth 64:59-63. Medline

Mahmood F, Mozere M, Zdebik AA, Stanescu HC, Tobin J, Beales PL, Kleta R, Bockenhauer D, Russell C (2013) Generation and validation of a zebrafish model of EAST (epilepsy, ataxia, sensorineural deafness and tubulopathy) syndrome. Dis Model Mech 6:652-660. CrossRef Medline

Masimirembwa CM, Thompson R, Andersson TB (2001) In vitro high throughput screening of compounds for favorable metabolic properties in drug discovery. Comb Chem High Throughput Screen 4:245-263. Medline

Novak AE, Jost MC, Lu Y, Taylor AD, Zakon HH, Ribera AB (2006) Gene duplications and evolution of vertebrate voltage-gated sodium channels. J Mol Evol 63:208-221. CrossRef Medline

Ottman R, Risch N (2012) Genetic epidemiology and gene discovery in epilepsy. In: Jasper's basic mechanisms of the epilepsies (Noebels JL, Avoli M, Rogawski M, Olsen R, Delgado-Escueta A, eds). New York: Oxford UP. pp. 651-658.

Raftery TD, Isales GM, Yozzo KL, Volz DC (2014) High-content screening assay for identification of chemicals impacting spontaneous activity in zebrafish embryos. Environ Sci Technol 48:804810. CrossRef Medline

Rothman RB, Baumann MH, Blough BE, Jacobson AE, Rice KC, Partilla JS (2010) Evidence for noncompetitive modulation of substrate-induced serotonin release. Synapse 64:862-869. CrossRef Medline

Saitoh M, Shinohara M, Hoshino H, Kubota M, Amemiya K, Takanashi JL, Hwang SK, Hirose S, Mizuguchi M (2012) Mutations of the SCN1A gene in acute encephalopathy. Epilepsia 53:558-564. CrossRef Medline

Schoonheim PJ, Arrenberg AB, Del Bene F, Baier H (2010) Optogenetic localization and genetic perturbation of saccade-generating neurons in zebrafish. J Neurosci 30:7111-7120. CrossRef Medline

Snowden M, Green DV (2008) The impact of diversity-based, highthroughput screening on drug discovery: "chance favours the prepared mind". Curr Opin Drug Discov Devel 11:553-558. Medline

Teng Y, Xie X, Walker S, Rempala G, Kozlowski DJ, Mumm JS, Cowell JK (2010) Knockdown of zebrafish Lgi1a results in abnormal development, brain defects and a seizure-like behavioral phenotype. Hum Mol Genet 19:4409-4420. CrossRef Medline

Tonduli LS, Testylier G, Masqueliez C, Lallement G, Monmaur P (2001) Effects of Huperzine used as pre-treatment against somaninduced seizures. Neurotoxicology 22:29-37. Medline

Wilmshurst JM, Berg AT, Lagae L, Newton CR, Cross JH (2014) The challenges and innovations for therapy in children with epilepsy. Nat Rev Neurol 10:249-260. CrossRef Medline

Winter MJ, Redfern WS, Hayfield AJ, Owen SF, Valentin JP, Hutchinson TH (2008) Validation of a larval zebrafish locomotor assay for assessing the seizure liability of early-stage development drugs. J Pharmacol Toxicol Methods 57:176-187. CrossRef Medline

Zhang YF, Gibbs JW 3rd, Coulter DA (1996) Anticonvulsant drug effects on spontaneous thalamocortical rhythms in vitro: ethosuximide, trimethadione, and dimethadione. Epilepsy Res 23:15-36. CrossRef 